ANNALES

POLONICI MATHEMATICI

$94.1(2008)$

\title{
Carathéodory solutions of hyperbolic functional differential inequalities with first order derivatives
}

\author{
by ADRIAN KARPOWICZ (Gdańsk)
}

Abstract. We consider the Darboux problem for a functional differential equation:

$$
\begin{aligned}
& \frac{\partial^{2} u}{\partial x \partial y}(x, y)=f\left(x, y, u_{(x, y)}, u(x, y), \frac{\partial u}{\partial x}(x, y), \frac{\partial u}{\partial y}(x, y)\right) \quad \text { a.e. in }[0, a] \times[0, b], \\
& u(x, y)=\psi(x, y) \quad \text { on }\left[-a_{0}, a\right] \times\left[-b_{0}, b\right] \backslash(0, a] \times(0, b],
\end{aligned}
$$

where the function $u_{(x, y)}:\left[-a_{0}, 0\right] \times\left[-b_{0}, 0\right] \rightarrow \mathbb{R}^{k}$ is defined by $u_{(x, y)}(s, t)=u(s+x, t+y)$ for $(s, t) \in\left[-a_{0}, 0\right] \times\left[-b_{0}, 0\right]$. We give a few theorems about weak and strong inequalities for this problem. We also discuss the case where the right-hand side of the differential equation is linear.

1. Introduction. Put $I=[0, a] \times[0, b], D=\left[-a_{0}, 0\right] \times\left[-b_{0}, 0\right]$, $I^{*}=\left[-a_{0}, a\right] \times\left[-b_{0}, b\right], I_{0}=\overline{I^{*} \backslash I}$. We always assume that $a, b>0$ and $a_{0}, b_{0} \in \mathbb{R}_{+}$, where $\mathbb{R}_{+}=[0,+\infty)$. We denote by $C\left(D, \mathbb{R}^{k}\right)$ and $L^{1}\left(D, \mathbb{R}^{k}\right)$ the spaces of continuous functions and of Lebesgue integrable functions from $D$ into $\mathbb{R}^{k}$, respectively. The symbol $|\cdot|$ denotes the maximum norm in $\mathbb{R}^{k}$. Moreover, $\|w\|_{0}$ denotes the usual supremum norm of $w \in C\left(D, \mathbb{R}^{k}\right)$. The inequality $x<y$ in $\mathbb{R}^{k}$ means that $x_{i}<y_{i}$ for each $i \in\{1, \ldots, k\}$, and similarly for " $\geq$ ", " $>$ " and " $\leq$ ". A function $f=\left(f_{1}, \ldots, f_{k}\right): I \times C\left(D, \mathbb{R}^{k}\right) \times$ $\mathbb{R}^{k} \times \mathbb{R}^{k} \times \mathbb{R}^{k} \rightarrow \mathbb{R}^{k}$ of the variables $(x, y, \omega, \eta, \mu, \nu)$ is said to be quasimonotonically nondecreasing with respect to $\eta$ if each $f_{i}$ is nondecreasing with respect to every $\eta_{j}$ for $j \neq i$. This function is said to be nondecreasing with respect to the functional argument $\omega$ if the inequality $\omega_{1} \leq \omega_{2}$ implies that $f\left(x, y, \omega_{1}, \eta, \mu, \nu\right) \leq f\left(x, y, \omega_{2}, \eta, \mu, \nu\right)$. Here $\omega_{1} \leq \omega_{2}$ means that $\omega_{1}(s, t) \leq \omega_{2}(s, t)$ for all $(s, t) \in D$.

2000 Mathematics Subject Classification: 35L70, 35R10, 35R45.

Key words and phrases: functional differential inequalities, hyperbolic equations, Darboux problem, solutions in the sense of Carathéodory. 
We consider the linear problem

$$
\begin{aligned}
& \frac{\partial^{2} u}{\partial x \partial y}(x, y)=C(x, y) u(x, y)+M(x, y) \frac{\partial u}{\partial x}(x, y) \\
& \quad+N(x, y) \frac{\partial u}{\partial y}(x, y)+P(x, y) u_{(x, y)} \quad \text { a.e. in } I, \\
& u(x, y)=\psi(x, y) \quad \text { on } I_{0},
\end{aligned}
$$

where $P(x, y): C\left(D, \mathbb{R}^{k}\right) \rightarrow \mathbb{R}^{k}$ is a linear operator for every $(x, y) \in I$ and $C, M, N$ are square $k \times k$ matrices.

We also consider the nonlinear problem

$$
\begin{aligned}
& \frac{\partial^{2} u}{\partial x \partial y}(x, y)=f\left(x, y, u_{(x, y)}, u(x, y), \frac{\partial u}{\partial x}(x, y), \frac{\partial u}{\partial y}(x, y)\right) \quad \text { a.e. in } I, \\
& u(x, y)=\psi(x, y) \quad \text { on } I_{0},
\end{aligned}
$$

where $f: I \times C\left(D, \mathbb{R}^{k}\right) \times \mathbb{R}^{k} \times \mathbb{R}^{k} \times \mathbb{R}^{k} \rightarrow \mathbb{R}^{k}$.

In both problems, $u_{(x, y)}: D \rightarrow \mathbb{R}^{k}$ is defined by the formula $u_{(x, y)}(s, t)=$ $u(s+x, t+y)$ for $(s, t) \in D$ and $\psi: I_{0} \rightarrow \mathbb{R}^{k}$ is a given continuous function. By a solution of the problem we mean an absolutely continuous function on $I$ and continuous on $I^{*}$ which has the derivatives $\partial u / \partial x, \partial u / \partial y, \partial^{2} u / \partial x \partial y$ almost everywhere on $I$, which satisfies the differential equation almost everywhere on $I$ and the initial condition everywhere on $I_{0}$. Now we give two examples of the operator $P(x, y)$.

EXAmple 1 . Let $\widetilde{P}(x, y)=\left(\widetilde{p}_{i j}(x, y)\right)_{i, j=1}^{k}$ be a square $k \times k$ matrix, where $\widetilde{p}_{i j} \in L^{1}(I, \mathbb{R}), \gamma_{1}: I \rightarrow\left[-a_{0}, a\right], \gamma_{2}: I \rightarrow\left[-b_{0}, b\right]$ and $\left(\gamma_{1}(x, y)-\right.$ $\left.x, \gamma_{2}(x, y)-y\right) \in D$ for all $(x, y) \in I$. If for every $(x, y) \in I$, we define the operator $P(x, y): C\left(D, \mathbb{R}^{k}\right) \rightarrow \mathbb{R}^{k}$ by the formula

$$
P(x, y) w=\widetilde{P}(x, y) w\left(\gamma_{1}(x, y)-x, \gamma_{2}(x, y)-y\right),
$$

then

$$
P(x, y) u_{(x, y)}=\widetilde{P}(x, y) u\left(\gamma_{1}(x, y), \gamma_{2}(x, y)\right) .
$$

Consequently, as a special case of (1) we get the following equation with a deviated argument:

$$
\begin{aligned}
\frac{\partial^{2} u}{\partial x \partial y}(x, y)= & C(x, y) u(x, y)+M(x, y) \frac{\partial u}{\partial x}(x, y) \\
& +N(x, y) \frac{\partial u}{\partial y}(x, y)+\widetilde{P}(x, y) u\left(\gamma_{1}(x, y), \gamma_{2}(x, y)\right) \quad \text { a.e. in } I .
\end{aligned}
$$

If we want to get an integro-differential equation then for every $(x, y) \in I$, 
we define

$$
P(x, y) w=\widetilde{P}(x, y) \int_{-a_{0}}^{0} \int_{-b_{0}}^{0} w(s, t) d s d t,
$$

and consequently we have

$$
P(x, y) u_{(x, y)}=\widetilde{P}(x, y) \int_{x-a_{0}}^{x} \int_{y-b_{0}}^{y} u(s, t) d s d t .
$$

This paper is devoted to the study of hyperbolic functional differential inequalities for the Darboux problem. In each section we consider both the linear and nonlinear problems. For the nonlinear problem we always assume that $f$ satisfies the Lipschitz condition. In most of the presented theorems we consider the case of weak inequalities. In the last section we study strongly monotone flows. In [4] we considered a simpler Darboux problem, where $f$ was independent of $\partial u / \partial x$ and $\partial u / \partial y$. Theorems about ordinary differential inequalities can be found in [3], [5]-[9], and for hyperbolic inequalities in [1], [2], [4], [5], [9].

2. Weak inequalities. In this section we will be concerned with weak inequalities for the linear and nonlinear problems.

Theorem 1 (nonnegativity). Suppose that:

(I) For every $(x, y) \in I$ we have a linear map $P(x, y)$ from $C\left(D, \mathbb{R}^{k}\right)$ into $\mathbb{R}^{k}$ which is positive in the sense that $w \geq 0$ implies $P(x, y) w \geq 0$ for $(x, y) \in I$.

(II) $C(x, y)=\left(c_{i j}(x, y)\right)_{i, j=1}^{k}, M(x, y)=\left(m_{i j}(x, y)\right)_{i, j=1}^{k}, N(x, y)=$ $\left(n_{i j}(x, y)\right)_{i, j=1}^{k}$ are $k \times k$ matrices, where $c_{i j}, m_{i j}, n_{i j} \in L^{1}(I, \mathbb{R})$ and $m_{i j}(x, y), n_{i j}(x, y) \geq 0$ a.e. in $I$ for $i \neq j$. There is a function $l \in L^{1}(I, \mathbb{R})$ such that

$$
\begin{aligned}
& l(x, y) \geq 3 \int_{0}^{x} l(z, y) d z \int_{0}^{y} l(x, z) d z \quad \text { a.e. in } I, \\
& |P(x, y) w| \leq l(x, y)\|w\|_{0} \quad \text { and } \quad\left|c_{i j}(x, y)\right| \leq l(x, y) \quad \text { a.e. in } I, \\
& c_{i j}(x, y) \geq 2 \int_{0}^{x} l(z, y) d z \int_{0}^{y} l(x, z) d z \quad \text { a.e. in } I \text { for } i \neq j, \\
& c_{i i}(x, y) \geq-l(x, y)+3 \int_{0}^{x} l(z, y) d z \int_{0}^{y} l(x, z) d z \quad \text { a.e. in } I, \\
& \left|m_{i j}(x, y)\right| \leq \int_{0}^{x} l(z, y) d z, \quad\left|n_{i j}(x, y)\right| \leq \int_{0}^{y} l(x, z) d z \quad \text { a.e. in } I,
\end{aligned}
$$

where $i, j=1, \ldots, k$. 
(III) $u$ is a function absolutely continuous on $I$ and continuous on $I^{*}$; $\partial u / \partial x, \partial u / \partial y, \partial^{2} u / \partial x \partial y$ exist a.e. on $I ; \partial u / \partial x$ is continuous with respect to the second variable on $I$; and $\partial u / \partial y$ is continuous with respect to the first variable on $I$. Furthermore,

$$
\begin{aligned}
& \frac{\partial^{2} u}{\partial x \partial y}(x, y) \geq C(x, y) u(x, y)+M(x, y) \frac{\partial u}{\partial x}(x, y) \\
&+N(x, y) \frac{\partial u}{\partial y}(x, y)+P(x, y) u_{(x, y)} \quad \text { a.e. in } I, \\
& u(x, y) \geq 0 \text { on } I_{0}, \quad \frac{\partial u}{\partial x}(x, 0) \geq 0 \text { on }[0, a], \quad \frac{\partial u}{\partial y}(0, y) \geq 0 \text { on }[0, b] .
\end{aligned}
$$

Then $u(x, y) \geq 0$ on $I$.

Proof. Let $v(x, y)=e^{H(x, y)} u(x, y)$, where

$$
\begin{aligned}
& H(x, y)=\iint_{0}^{x} \int_{0}^{y} h\left(z_{1}, z_{2}\right) d z_{2} d z_{1} \quad \text { for }(x, y) \in I^{*}, \\
& h(x, y)= \begin{cases}l(x, y) & \text { for }(x, y) \in I \\
0 & \text { for }(x, y) \in I^{*} \backslash I .\end{cases}
\end{aligned}
$$

From (2) and

$$
\begin{aligned}
& \frac{\partial u}{\partial x}(x, y)=e^{-H(x, y)}\left\{\frac{\partial v}{\partial x}(x, y)-\int_{0}^{y} l(x, z) d z v(x, y)\right\} \\
& \frac{\partial u}{\partial y}(x, y)=e^{-H(x, y)}\left\{\frac{\partial v}{\partial y}(x, y)-\int_{0}^{x} l(z, y) d z v(x, y)\right\}
\end{aligned}
$$

we get

$$
\begin{aligned}
\frac{\partial^{2} v}{\partial x \partial y}(x, y) \geq & \widetilde{C}(x, y) v(x, y)+\widetilde{M}(x, y) \frac{\partial v}{\partial x}(x, y) \\
& +\widetilde{N}(x, y) \frac{\partial v}{\partial y}(x, y)+Q(x, y)\left(e^{-H} v\right)_{(x, y)}
\end{aligned}
$$

where

$$
\begin{aligned}
\widetilde{C}(x, y)= & C(x, y)+\left[l(x, y)-\int_{0}^{x} l(z, y) d z \int_{0}^{y} l(x, z) d z\right] E \\
& -\int_{0}^{y} l(x, z) d z M(x, y)-\int_{0}^{x} l(z, y) d z N(x, y), \\
\widetilde{M}(x, y)= & M(x, y)+\int_{0}^{x} l(z, y) d z E,
\end{aligned}
$$




$$
\begin{aligned}
\widetilde{N}(x, y) & =N(x, y)+\int_{0}^{y} l(x, z) d z E, \\
Q(x, y) w & =e^{H(x, y)} P(x, y) w,
\end{aligned}
$$

and $E$ denotes the unit matrix.

Note that $\widetilde{C}(x, y), \widetilde{M}(x, y), \widetilde{N}(x, y) \geq 0$, and that $Q(x, y)$ is a linear and positive operator from $C\left(D, \mathbb{R}^{k}\right)$ into $\mathbb{R}^{k}$ for every $(x, y) \in I$.

Define $\varrho: I^{*} \rightarrow \mathbb{R}$ by $\varrho(x, y)=e^{2(k+2) H(x, y)+x+y}$, and $r: I^{*} \rightarrow \mathbb{R}^{k}$ by $r(x, y)=(\varrho(x, y), \ldots, \varrho(x, y))$. Then

$$
\begin{gathered}
\frac{\partial^{2} r}{\partial x \partial y}(x, y)=2(k+2) l(x, y) r(x, y)+4(k+2)^{2} \int_{0}^{x} l(z, y) d z \int_{0}^{y} l(x, z) d z r(x, y) \\
+2(k+2) \int_{0}^{x} l(z, y) d z r(x, y)+2(k+2) \int_{0}^{y} l(x, z) d z r(x, y)+r(x, y), \\
Q(x, y)\left(e^{-H} r\right)_{(x, y)}=e^{H(x, y)} P(x, y)\left(e^{-H} r\right)_{(x, y)} .
\end{gathered}
$$

Using suitable estimates for $\widetilde{C}(x, y), \widetilde{M}(x, y), \widetilde{N}(x, y)$ and $P(x, y)$, we get

$$
\begin{aligned}
0 \leq \widetilde{C}(x, y) r(x, y) \leq\{ & \left.(k+1) l(x, y)+(2 k-1) \int_{0}^{x} l(z, y) d z \int_{0}^{y} l(x, z) d z\right\} r(x, y), \\
0 \leq \widetilde{M}(x, y) \frac{\partial r}{\partial x}(x, y) \leq & \left\{2(k+2)(k+1) \int_{0}^{x} l(z, y) d z \int_{0}^{y} l(x, z) d z\right. \\
& \left.+(k+1) \int_{0}^{x} l(z, y) d z\right\} r(x, y) \\
0 \leq \widetilde{N}(x, y) \frac{\partial r}{\partial y}(x, y) \leq & \left\{2(k+2)(k+1) \int_{0}^{x} l(z, y) d z \int_{0}^{y} l(x, z) d z\right. \\
& \left.+(k+1) \int_{0}^{y} l(x, z) d z\right\} r(x, y), \\
\left|Q(x, y)\left(e^{-H} r\right)_{(x, y)}\right| \leq & e^{H(x, y)} l(x, y)\left\|e^{(2 k+3) H(x, y)+x+y}\right\|_{0} \leq l(x, y) r(x, y) .
\end{aligned}
$$

Thus

$$
\begin{aligned}
& \widetilde{C}(x, y) r(x, y)+\widetilde{M}(x, y) \frac{\partial r}{\partial x}(x, y)+\widetilde{N}(x, y) \frac{\partial r}{\partial y}(x, y)+Q(x, y)\left(e^{-H} r\right)_{(x, y)} \\
& \leq(k+2) l(x, y) r(x, y)+\left(4 k^{2}+14 k+7\right) \int_{0}^{x} l(z, y) d z \int_{0}^{y} l(x, z) d z r(x, y) \\
& \quad+(k+1) \int_{0}^{x} l(z, y) d z r(x, y)+(k+1) \int_{0}^{y} l(x, z) d z r(x, y) \leq \frac{\partial^{2} r}{\partial x \partial y}(x, y) .
\end{aligned}
$$


For each $\varepsilon>0$, define $v_{\varepsilon}(x, y)=v(x, y)+\varepsilon r(x, y)$. From linearity of $Q(x, y)$ we obtain

$$
\begin{aligned}
\frac{\partial^{2} v_{\varepsilon}}{\partial x \partial y}(x, y) \geq & \widetilde{C}(x, y) v_{\varepsilon}(x, y)+\widetilde{M}(x, y) \frac{\partial v_{\varepsilon}}{\partial x}(x, y) \\
& +\widetilde{N}(x, y) \frac{\partial v_{\varepsilon}}{\partial y}(x, y)+Q(x, y)\left(e^{-H} v_{\varepsilon}\right)_{(x, y)} .
\end{aligned}
$$

Since $v_{\varepsilon}(x, y)=v(x, y)+\varepsilon r(x, y)$, we have

$$
\begin{aligned}
\frac{\partial v_{\varepsilon}}{\partial x}(x, y)= & e^{H(x, y)} \frac{\partial u}{\partial x}(x, y)+\int_{0}^{y} l(x, z) d z e^{H(x, y)} u(x, y) \\
& +\varepsilon\left[2(k+2) \int_{0}^{y} l(x, z) d z+1\right] r(x, y), \\
\frac{\partial v_{\varepsilon}}{\partial y}(x, y)= & e^{H(x, y)} \frac{\partial u}{\partial y}(x, y)+\int_{0}^{x} l(z, y) d z e^{H(x, y)} u(x, y) \\
& +\varepsilon\left[2(k+2) \int_{0}^{x} l(z, y) d z+1\right] r(x, y) .
\end{aligned}
$$

Note that

$$
v_{\varepsilon}(x, y)>0 \text { on } I_{0}, \quad \frac{\partial v_{\varepsilon}}{\partial x}(x, 0)>0 \text { on }[0, a], \quad \frac{\partial v_{\varepsilon}}{\partial y}(0, y)>0 \text { on }[0, b] .
$$

Since $v_{\varepsilon}$ is continuous and $\partial v_{\varepsilon} / \partial x$ is continuous with respect to the second variable and $\partial v_{\varepsilon} / \partial y$ is continuous with respect to the first variable on $I$, we see that there exists $c>0$ such that

$$
\begin{array}{ll}
v_{\varepsilon}(x, y)>0 & \text { on } I_{c}=I^{*} \backslash(c, a] \times(c, b], \\
\frac{\partial v_{\varepsilon}}{\partial x}(x, y)>0 & \text { on }[0, a] \times[0, c], \\
\frac{\partial v_{\varepsilon}}{\partial y}(x, y)>0 & \text { on }[0, c] \times[0, b] .
\end{array}
$$

Thus all terms on the right hand side of $(3)$ are nonnegative on $[0, c] \times[0, c]$. Therefore $\partial^{2} v_{\varepsilon} / \partial x \partial y \geq 0$ on $I \backslash(c, a] \times(c, b]$. Integrating this inequality with respect to $y$ we see that $\partial v_{\varepsilon} / \partial x$ is nondecreasing with respect to the second variable. Similarly, $\partial v_{\varepsilon} / \partial y$ is nondecreasing with respect to the first variable on $[0, c] \times[0, c]$. Next we find that $v_{\varepsilon}, \partial v_{\varepsilon} / \partial x, \partial v_{\varepsilon} / \partial y$ are nonnegative and nondecreasing with respect to appropriate variables on $I_{c} \backslash\left(I^{*} \backslash I\right)$. We continue in this fashion to deduce that $v_{\varepsilon}, \partial v_{\varepsilon} / \partial x, \partial v_{\varepsilon} / \partial y$ are nonnegative and nondecreasing with respect to appropriate variables on $I$. Letting $\varepsilon \rightarrow 0$ we conclude that $v \geq 0$, which implies that $u \geq 0$ on $I$. 
Remark 1. Since $v_{\varepsilon}, \partial v_{\varepsilon} / \partial x, \partial v_{\varepsilon} / \partial y>0$ on $I$, we have $v \geq 0$ on $I$ and $\partial v / \partial x, \partial v / \partial y \geq 0$ a.e. on $I$. Furthermore, $v$ is absolutely continuous, which implies that it is nondecreasing with respect to the first and second variables. Since $u(x, y)=e^{-H(x, y)} v(x, y)$ it follows that there are disjoint index sets $\alpha$ and $\beta$ such that $\alpha \cup \beta=\{1, \ldots, k\}$ and $u_{i}(x, y)>0$ on $I^{*} \backslash I_{0}$ for $i \in \alpha$ and $u_{j}(x, y)=0$ on $A_{j}, u_{j}(x, y)>0$ on $B_{j}$ for $j \in \beta$. Here $A_{j}$ and $B_{j}$ are disjoint sets such that $A_{j} \cup B_{j}=I$ and $\left[\mathbb{R}_{+}^{2}+\left(x_{0}, y_{0}\right)\right] \cap I \subset B_{j}$ for each $\left(x_{0}, y_{0}\right) \in \bar{A}_{j} \cap \bar{B}_{j}$.

REMARK 2. From the proof of Theorem 1 it follows that

$$
\begin{array}{ll}
\frac{\partial u}{\partial x}(x, y)+\int_{0}^{y} l(x, z) d z u(x, y) \geq 0 & \text { a.e. in } I, \\
\frac{\partial u}{\partial y}(x, y)+\int_{0}^{x} l(z, y) d z u(x, y) \geq 0 & \text { a.e. in } I .
\end{array}
$$

THEOREM 2 (weak inequalities). Suppose that:

(I) The function $f: I \times C\left(D, \mathbb{R}^{k}\right) \times \mathbb{R}^{k} \times \mathbb{R}^{k} \times \mathbb{R}^{k} \rightarrow \mathbb{R}^{k}$ of the variables $(x, y, \omega, \eta, \mu, \nu)$ is nondecreasing with respect to $\omega, \mu, \nu$ and quasimonotone nondecreasing with respect to $\eta$.

(II) For each $A>0$ there exists a function $l \in L^{1}(I, \mathbb{R})$ such that

$$
l(x, y) \geq 3 \int_{0}^{x} l(z, y) d z \int_{0}^{y} l(x, z) d z \quad \text { a.e. on } I,
$$

$$
\left|f_{i}(x, y, \bar{\omega}, \bar{\eta}, \bar{\mu}, \bar{\nu})-f_{i}(x, y, \omega, \eta, \mu, \nu)\right| \leq l(x, y)\left(\|\bar{\omega}-\omega\|_{0}+|\bar{\eta}-\eta|\right)
$$

$$
+\int_{0}^{x} l(z, y) d z|\bar{\mu}-\mu|+\int_{0}^{y} l(x, z) d z|\bar{\nu}-\nu|,
$$

$$
f_{i}(x, y, \omega, \bar{\eta}, \mu, \nu)-f_{i}(x, y, \omega, \eta, \mu, \nu)
$$

$$
\begin{aligned}
\geq & {\left[-l(x, y)+3 \int_{0}^{x} l(z, y) d z \int_{0}^{y} l(x, z) d z\right]\left(\bar{\eta}_{i}-\eta_{i}\right) } \\
& +2 \int_{0}^{x} l(z, y) d z \int_{0}^{y} l(x, z) d z|\bar{\eta}-\eta|_{i},
\end{aligned}
$$

for $\|\omega\|_{0},\|\bar{\omega}\|_{0},|\eta|,|\bar{\eta}| \leq A$ and $\eta \leq \bar{\eta}$, where $|u|_{i}=\max \left\{\left|u_{j}\right|: j \neq i\right\}$.

(III) $w, v$ are functions absolutely continuous on I and continuous on $I^{*}$; $\partial w / \partial x, \partial v / \partial x, \partial w / \partial y, \partial v / \partial y, \partial^{2} w / \partial x \partial y, \partial^{2} v / \partial x \partial y$ exist a.e. on $I ; \partial w / \partial x, \partial v / \partial x$ are continuous with respect to the second variable on $I$; and $\partial w / \partial y, \partial v / \partial y$ are continuous with respect to the first variable on $I$. Furthermore, 


$$
\begin{array}{ll}
\frac{\partial^{2} v}{\partial x \partial y}(x, y) \leq f\left(x, y, v_{(x, y)}, v(x, y), \frac{\partial v}{\partial x}(x, y), \frac{\partial v}{\partial y}(x, y)\right) \quad \text { and } \\
\frac{\partial^{2} w}{\partial x \partial y}(x, y) \geq f\left(x, y, w_{(x, y)}, w(x, y), \frac{\partial w}{\partial x}(x, y), \frac{\partial w}{\partial y}(x, y)\right) \quad \text { a.e. in } I, \\
v(x, y) \leq w(x, y) \quad \text { on } I_{0}, \\
\frac{\partial v}{\partial x}(x, 0) \leq \frac{\partial w}{\partial x}(x, 0) \quad \text { on }[0, a], \quad \frac{\partial v}{\partial y}(0, y) \leq \frac{\partial w}{\partial y}(0, y) \quad \text { on }[0, b] .
\end{array}
$$

Then $v(x, y) \leq w(x, y)$ on $I$.

Proof. Suppose $|v(x, y)|,|w(x, y)| \leq A-1$ for all $(x, y) \in I$ and $l$ be the function in (II) corresponding to $A$. Define $V(x, y)=e^{H(x, y)} v(x, y)$ and $W(x, y)=e^{H(x, y)} w(x, y)$, where $H(x, y)$ is as in the proof of Theorem 1 . Then

$$
\begin{aligned}
\frac{\partial^{2} V}{\partial x \partial y}(x, y) \leq & e^{H(x, y)} f\left(x, y, v_{(x, y)}, v(x, y), \frac{\partial v}{\partial x}(x, y), \frac{\partial v}{\partial y}(x, y)\right) \\
& +e^{H(x, y)}\left[l(x, y)+\int_{0}^{x} l(z, y) d z \int_{0}^{y} l(x, z) d z\right] v(x, y) \\
& +e^{H(x, y)} \int_{0}^{x} l(z, y) d z \frac{\partial v}{\partial x}(x, y)+e^{H(x, y)} \int_{0}^{y} l(x, z) d z \frac{\partial v}{\partial y}(x, y) .
\end{aligned}
$$

From

$$
\begin{aligned}
& \frac{\partial v}{\partial x}(x, y)=e^{-H(x, y)}\left(\frac{\partial V}{\partial x}(x, y)-\int_{0}^{y} l(x, z) d z V(x, y)\right) \\
& \frac{\partial v}{\partial y}(x, y)=e^{-H(x, y)}\left(\frac{\partial V}{\partial y}(x, y)-\int_{0}^{x} l(z, y) d z V(x, y)\right)
\end{aligned}
$$

we have

where

$$
\frac{\partial^{2} V}{\partial x \partial y}(x, y) \leq e^{H(x, y)}(G v)(x, y)
$$

$$
\begin{aligned}
(G v)(x, y)= & f\left(x, y, v_{(x, y)}, v(x, y), \frac{\partial v}{\partial x}(x, y), \frac{\partial v}{\partial y}(x, y)\right)+l_{1}(x, y) v(x, y) \\
& +e^{-H(x, y)} l_{2}(x, y) \frac{\partial V}{\partial x}(x, y)+e^{-H(x, y)} l_{3}(x, y) \frac{\partial V}{\partial y}(x, y),
\end{aligned}
$$

with

$$
\begin{aligned}
& l_{1}(x, y)=l(x, y)-\int_{0}^{x} l(z, y) d z \int_{0}^{y} l(x, z) d z, \\
& l_{2}(x, y)=\int_{0}^{x} l(z, y) d z, \quad l_{3}(x, y)=\int_{0}^{y} l(x, z) d z .
\end{aligned}
$$


Similarly, we get

$$
\frac{\partial^{2} W}{\partial x \partial y}(x, y) \geq e^{H(x, y)}(G w)(x, y),
$$

where $(G w)(x, y)$ is defined by a formula similar to that for $(G v)(x, y)$.

Fix an arbitrary point $\left(x_{0}, y_{0}\right) \in I$. We will show that if

$$
v(x, y) \leq w(x, y) \quad \text { for }(x, y) \in\left[x_{0}-a_{0}, x_{0}\right] \times\left[y_{0}-b_{0}, y_{0}\right]
$$

and

$$
\frac{\partial V}{\partial x}\left(x_{0}, y_{0}\right) \leq \frac{\partial W}{\partial x}\left(x_{0}, y_{0}\right) \quad \text { and } \quad \frac{\partial V}{\partial y}\left(x_{0}, y_{0}\right) \leq \frac{\partial W}{\partial y}\left(x_{0}, y_{0}\right)
$$

then

$$
(G v)\left(x_{0}, y_{0}\right) \leq(G w)\left(x_{0}, y_{0}\right) .
$$

Let $T$ denote the index set such that

$$
\frac{\partial v_{j}}{\partial x}\left(x_{0}, y_{0}\right) \leq \frac{\partial w_{j}}{\partial x}\left(x_{0}, y_{0}\right) \quad \text { for } j \in T,
$$

and $R$ denote the index set such that

$$
\frac{\partial v_{j}}{\partial y}\left(x_{0}, y_{0}\right) \leq \frac{\partial w_{j}}{\partial y}\left(x_{0}, y_{0}\right) \quad \text { for } j \in R .
$$

Furthermore, set $T^{\prime}=\{1, \ldots, k\} \backslash T$ and $R^{\prime}=\{1, \ldots, k\} \backslash R$. Some of these sets may be empty. Let $\left(w^{T}\left(x_{0}, y_{0}\right), v^{T^{\prime}}\left(x_{0}, y_{0}\right)\right) \in \mathbb{R}^{k}$ denote the vector whose $j$ th coordinate is $w_{j}\left(x_{0}, y_{0}\right)$ if $j \in T$, and $v_{j}\left(x_{0}, y_{0}\right)$ if $j \in T^{\prime}$. Moreover, $|u|_{S}=\max \left\{\left|u_{j}\right|: j \in S\right\}$, where $S$ is some index set.

From (6) it follows that

$$
\begin{aligned}
& \frac{\partial v}{\partial x}\left(x_{0}, y_{0}\right)-\frac{\partial w}{\partial x}\left(x_{0}, y_{0}\right) \leq \int_{0}^{y_{0}} l\left(x_{0}, z\right) d z\left(w\left(x_{0}, y_{0}\right)-v\left(x_{0}, y_{0}\right)\right), \\
& \frac{\partial v}{\partial y}\left(x_{0}, y_{0}\right)-\frac{\partial w}{\partial y}\left(x_{0}, y_{0}\right) \leq \int_{0}^{x_{0}} l\left(z, y_{0}\right) d z\left(w\left(x_{0}, y_{0}\right)-v\left(x_{0}, y_{0}\right)\right) .
\end{aligned}
$$

Therefore for $j \in T^{\prime}$ and $k \in R^{\prime}$ we have

$$
\begin{aligned}
\left|\frac{\partial v_{j}}{\partial x}\left(x_{0}, y_{0}\right)-\frac{\partial w_{j}}{\partial x}\left(x_{0}, y_{0}\right)\right| & \leq \int_{0}^{y_{0}} l\left(x_{0}, z\right) d z\left(w_{j}\left(x_{0}, y_{0}\right)-v_{j}\left(x_{0}, y_{0}\right)\right), \\
\left|\frac{\partial v_{k}}{\partial y}\left(x_{0}, y_{0}\right)-\frac{\partial w_{k}}{\partial y}\left(x_{0}, y_{0}\right)\right| & \leq \int_{0}^{x_{0}} l\left(z, y_{0}\right) d z\left(w_{k}\left(x_{0}, y_{0}\right)-v_{k}\left(x_{0}, y_{0}\right)\right) .
\end{aligned}
$$

In order to prove $(6) \Rightarrow(7)$ we will use the monotonicity of $f$ with respect to $\omega, \mu, \nu$, quasimonotonicity of $f$ with respect to $\eta$, assumptions (4), (5), and 
inequalities (8), (9). Then

$$
\begin{aligned}
& (G v)_{i}\left(x_{0}, y_{0}\right) \\
& \leq f_{i}\left(x_{0}, y_{0}, w_{\left(x_{0}, y_{0}\right)}, v\left(x_{0}, y_{0}\right), \frac{\partial\left(w^{T}, v^{T^{\prime}}\right)}{\partial x}\left(x_{0}, y_{0}\right), \frac{\partial\left(w^{R}, v^{R^{\prime}}\right)}{\partial y}\left(x_{0}, y_{0}\right)\right) \\
& +\left(l\left(x_{0}, y_{0}\right)-\int_{0}^{x_{0}} l\left(z, y_{0}\right) d z \int_{0}^{y_{0}} l\left(x_{0}, z\right) d z\right) v_{i}\left(x_{0}, y_{0}\right) \\
& +e^{-H\left(x_{0}, y_{0}\right)} l_{2}\left(x_{0}, y_{0}\right) \frac{\partial W_{i}}{\partial x}\left(x_{0}, y_{0}\right)+e^{-H\left(x_{0}, y_{0}\right)} l_{3}\left(x_{0}, y_{0}\right) \frac{\partial W_{i}}{\partial y}\left(x_{0}, y_{0}\right) \\
& \leq f_{i}\left(x_{0}, y_{0}, w_{\left(x_{0}, y_{0}\right)}, w\left(x_{0}, y_{0}\right), \frac{\partial w}{\partial x}\left(x_{0}, y_{0}\right), \frac{\partial w}{\partial y}\left(x_{0}, y_{0}\right)\right) \\
& +\left(l\left(x_{0}, y_{0}\right)-3 \int_{0}^{x_{0}} l\left(z, y_{0}\right) d z \int_{0}^{y_{0}} l\left(x_{0}, z\right) d z\right) w_{i}\left(x_{0}, y_{0}\right) \\
& +2 \int_{0}^{x_{0}} l\left(z, y_{0}\right) d z \int_{0}^{y_{0}} l\left(x_{0}, z\right) d z v_{i}\left(x_{0}, y_{0}\right) \\
& -2 \int_{0}^{x_{0}} l\left(z, y_{0}\right) d z \int_{0}^{y_{0}} l\left(x_{0}, z\right) d z\left|w\left(x_{0}, y_{0}\right)-v\left(x_{0}, y_{0}\right)\right|_{i} \\
& +\int_{0}^{x_{0}} l\left(z, y_{0}\right) d z\left|\frac{\partial v}{\partial x}\left(x_{0}, y_{0}\right)-\frac{\partial w}{\partial x}\left(x_{0}, y_{0}\right)\right|_{T^{\prime}} \\
& +\int_{0}^{y_{0}} l\left(x_{0}, z\right) d z\left|\frac{\partial v}{\partial y}\left(x_{0}, y_{0}\right)-\frac{\partial w}{\partial y}\left(x_{0}, y_{0}\right)\right|_{R^{\prime}} \\
& +e^{-H\left(x_{0}, y_{0}\right)} l_{2}\left(x_{0}, y_{0}\right) \frac{\partial W_{i}}{\partial x}\left(x_{0}, y_{0}\right)+e^{-H\left(x_{0}, y_{0}\right)} l_{3}\left(x_{0}, y_{0}\right) \frac{\partial W_{i}}{\partial y}\left(x_{0}, y_{0}\right) \\
& \leq f_{i}\left(x_{0}, y_{0}, w_{\left(x_{0}, y_{0}\right)}, w\left(x_{0}, y_{0}\right), \frac{\partial w}{\partial x}\left(x_{0}, y_{0}\right), \frac{\partial w}{\partial y}\left(x_{0}, y_{0}\right)\right)+l_{1}\left(x, y_{0}\right) w_{i}\left(x_{0}, y_{0}\right) \\
& -2 \int_{0}^{x_{0}} l\left(z, y_{0}\right) d z \int_{0}^{y_{0}} l\left(x_{0}, z\right) d z\left|w\left(x_{0}, y_{0}\right)-v\left(x_{0}, y_{0}\right)\right| \\
& +\int_{0}^{x_{0}} l\left(z, y_{0}\right) d z \int_{0}^{y_{0}} l\left(x_{0}, z\right) d z\left|w\left(x_{0}, y_{0}\right)-v\left(x_{0}, y_{0}\right)\right|_{T^{\prime}} \\
& +\int_{0}^{y_{0}} l\left(x_{0}, z\right) d z \int_{0}^{x_{0}} l\left(z, y_{0}\right) d z\left|w\left(x_{0}, y_{0}\right)-v\left(x_{0}, y_{0}\right)\right|_{R^{\prime}} \\
& +e^{-H\left(x_{0}, y_{0}\right)} l_{2}\left(x_{0}, y_{0}\right) \frac{\partial W_{i}}{\partial x}\left(x_{0}, y_{0}\right)+e^{-H(x, y)} l_{3}\left(x_{0}, y_{0}\right) \frac{\partial W_{i}}{\partial y}\left(x_{0}, y_{0}\right) \\
& \leq(G w)_{i}\left(x_{0}, y_{0}\right)
\end{aligned}
$$


Define $\varrho: I^{*} \rightarrow \mathbb{R}$ by $\varrho(x, y)=e^{3 H(x, y)+x+y}$ and $r, R: I^{*} \rightarrow \mathbb{R}^{k}$ by $r(x, y)=(\varrho(x, y), \ldots, \varrho(x, y)), R(x, y)=e^{H(x, y)} r(x, y)$. Defining $w_{\varepsilon}(x, y)$ $=w(x, y)+\varepsilon r(x, y)$ and $W_{\varepsilon}(x, y)=e^{H(x, y)} w_{\varepsilon}(x, y)=W(x, y)+\varepsilon R(x, y)$ we have

$$
\begin{aligned}
\frac{\partial^{2} W_{\varepsilon}}{\partial x \partial y}(x, y) \geq & e^{H(x, y)}(G w)(x, y)+\varepsilon e^{H(x, y)}\{4 l(x, y) r(x, y) \\
& +16 \int_{0}^{x} l(z, y) d z \int_{0}^{y} l(x, z) d z r(x, y)+4 \int_{0}^{x} l(z, y) d z r(x, y) \\
& \left.+4 \int_{0}^{y} l(x, z) d z r(x, y)+r(x, y)\right\}
\end{aligned}
$$

We can choose $\varepsilon>0$ sufficiently small in order that $\left|\left(w_{\varepsilon}\right)_{i}(x, y)\right| \leq A$ for each $i \in\{1, \ldots, k\}$. Thus

$$
\begin{aligned}
& \left|\left(G w_{\varepsilon}\right)_{i}(x, y)-(G w)_{i}(x, y)\right| \\
& =\mid f_{i}\left(x, y,\left(w_{\varepsilon}\right)_{(x, y)}, w_{\varepsilon}(x, y), \frac{\partial w_{\varepsilon}}{\partial x}(x, y), \frac{\partial w_{\varepsilon}}{\partial y}(x, y)\right) \\
& \quad-f_{i}\left(x, y, w_{(x, y)}, w(x, y), \frac{\partial w}{\partial x}(x, y), \frac{\partial w}{\partial y}(x, y)\right)+\varepsilon l_{1}(x, y) r_{i}(x, y) \\
& \quad+\varepsilon e^{-H(x, y)} l_{2}(x, y) \frac{\partial R_{i}}{\partial x}(x, y)+\varepsilon e^{-H(x, y)} l_{3}(x, y) \frac{\partial R_{i}}{\partial y}(x, y) \mid \\
& \leq l(x, y)\left(\|\varepsilon r\|_{0}+|\varepsilon r|\right)+\int_{0}^{x} l(z, y) d z\left|\varepsilon \frac{\partial r}{\partial x}(x, y)\right| \\
& \quad+\int_{0}^{y} l(x, z) d z\left|\varepsilon \frac{\partial r}{\partial y}(x, y)\right|+\varepsilon l_{1}(x, y) \varrho(x, y)+e^{-H(x, y)} \varepsilon l_{2}(x, y)\left|\frac{\partial R_{i}}{\partial x}(x, y)\right| \\
& \quad+e^{-H(x, y)} \varepsilon l_{3}(x, y)\left|\frac{\partial R_{i}}{\partial y}(x, y)\right| \leq 2 \varepsilon l(x, y) \varrho(x, y) \\
& \quad+\varepsilon l_{2}(x, y)\left[3 l_{3}(x, y)+1\right] \varrho(x, y)+\varepsilon l_{3}(x, y)\left[3 h_{2}(x, y)+1\right] \varrho(x, y)+\varepsilon l_{1} \varrho(x, y) \\
& \quad+\varepsilon l_{2}(x, y)\left[4 l_{3}(x, y)+1\right] \varrho(x, y)+\varepsilon l_{3}(x, y)\left[4 l_{2}(x, y)+1\right] \varrho(x, y) \\
& =3 \varepsilon l(x, y) \varrho(x, y)+13 \varepsilon \int_{0}^{x} l(z, y) d z \int_{0}^{y} l(x, z) d z \varrho(x, y) \\
& \quad+\varepsilon \int_{0}^{x} l(z, y) d z \varrho(x, y)+\varepsilon \int_{0}^{y} l(x, z) d z \varrho(x, y) .
\end{aligned}
$$


From the above inequality we get

$$
\begin{aligned}
e^{H(x, y)}\left(G w_{\varepsilon}\right)(x, y) \leq & e^{H(x, y)}(G w)(x, y) \\
& +\varepsilon e^{H(x, y)}\{3 l(x, y) r(x, y) \\
& +13 \int_{0}^{x} l(z, y) d z \int_{0}^{y} l(x, z) d z r(x, y) \\
& \left.+\int_{0}^{x} l(z, y) d z r(x, y)+\int_{0}^{y} l(x, z) d z r(x, y)\right\} \\
\leq & \frac{\partial^{2} W_{\varepsilon}}{\partial x \partial y}(x, y) .
\end{aligned}
$$

We note that

$$
\begin{array}{ll}
w_{\varepsilon}(x, y)>w(x, y) \geq v(x, y) & \text { on } I_{0}, \\
\frac{\partial w_{\varepsilon}}{\partial x}(x, 0)>\frac{\partial w}{\partial x}(x, 0) \geq \frac{\partial v}{\partial x}(x, 0) & \text { on }[0, a], \\
\frac{\partial w_{\varepsilon}}{\partial y}(0, y)>\frac{\partial w}{\partial y}(0, y) \geq \frac{\partial v}{\partial y}(0, y) & \text { on }[0, b] .
\end{array}
$$

Therefore there exists $c>0$ such that

$$
\begin{array}{ll}
w_{\varepsilon}(x, y)>v(x, y) & \text { on } I_{c}=I^{*} \backslash(c, a] \times(c, b], \\
\frac{\partial w_{\varepsilon}}{\partial x}(x, y)>\frac{\partial v}{\partial x}(x, y) & \text { on }[0, a] \times[0, c], \\
\frac{\partial w_{\varepsilon}}{\partial y}(x, y)>\frac{\partial v}{\partial y}(x, y) & \text { on }[0, c] \times[0, b] .
\end{array}
$$

From (11) and (12) we have

$$
\frac{\partial W_{\varepsilon}}{\partial x}(x, y)>\frac{\partial V}{\partial x}(x, y) \quad \text { on }[0, a] \times[0, c] .
$$

From (11) and (13) we get

$$
\frac{\partial W_{\varepsilon}}{\partial y}(x, y)>\frac{\partial V}{\partial y}(x, y) \quad \text { on }[0, c] \times[0, b] .
$$

Thus

$$
\begin{gathered}
w_{\varepsilon}(x, y)>v(x, y) \quad \text { on } I_{c} \cup I_{0}, \\
\frac{\partial W_{\varepsilon}}{\partial x}(x, y)>\frac{\partial V}{\partial x}(x, y) \quad \text { and } \quad \frac{\partial W_{\varepsilon}}{\partial y}(x, y)>\frac{\partial V}{\partial y}(x, y) \quad \text { on }[0, c] \times[0, c] .
\end{gathered}
$$

Taking an arbitrary point $\left(x_{0}, y_{0}\right) \in[0, c] \times[0, c]$ we deduce from $(7),(10)$ 
the inequality

$$
\begin{aligned}
\frac{\partial^{2} W_{\varepsilon}}{\partial x \partial y}\left(x_{0}, y_{0}\right) & \geq e^{H\left(x_{0}, y_{0}\right)}\left(G w_{\varepsilon}\right)\left(x_{0}, y_{0}\right) \geq e^{H\left(x_{0}, y_{0}\right)}(G v)\left(x_{0}, y_{0}\right) \\
& \geq \frac{\partial^{2} V}{\partial x \partial y}\left(x_{0}, y_{0}\right)
\end{aligned}
$$

Thus

$$
\frac{\partial^{2}}{\partial x \partial y}\left(W_{\varepsilon}-V\right)(x, y) \geq 0 \quad \text { on }[0, c] \times[0, c]
$$

Similarly to the proof of Theorem 1, from the continuity of $\partial w / \partial x, \partial v / \partial x$, $\partial r / \partial x$ with respect to the second variable, and $\partial w / \partial y, \partial v / \partial y, \partial r / \partial y$ with respect to the first variable, and from (14), we see that $\frac{\partial}{\partial x}\left(W_{\varepsilon}-V\right)$ is nondecreasing with respect to the second variable, and $\frac{\partial}{\partial y}\left(W_{\varepsilon}-V\right)$ is nondecreasing with respect to the first variable on $[0, c] \times[0, c]$. Therefore $\frac{\partial}{\partial x}\left(W_{\varepsilon}-V\right)$ and $\frac{\partial}{\partial y}\left(W_{\varepsilon}-V\right)$ remain positive on $I_{c}$. Repeating this reasoning for the set $I_{c}$ we find that $W_{\varepsilon}-V, \frac{\partial}{\partial x}\left(W_{\varepsilon}-V\right), \frac{\partial}{\partial y}\left(W_{\varepsilon}-V\right)$ are positive on $I$. Thus $W_{\varepsilon}>V$ on $I$. Letting $\varepsilon \rightarrow 0$ we get $W \geq V$ on $I$, which implies $w \geq v$ on $I$.

REMARK 3. Analysis similar to that in Remark 1 shows that there are two disjoint index sets $\alpha$ and $\beta$ such that $\alpha \cup \beta=\{1, \ldots, k\}, w_{i}(x, y)>$ $v_{i}(x, y)$ on $I^{*} \backslash I_{0}$ for $i \in \alpha, w_{j}(x, y)=v_{j}(x, y)$ on $A_{j}$, and $w_{j}(x, y)>v_{j}(x, y)$ on $B_{j}$ for $j \in \beta$, where $A_{j}$ and $B_{j}$ are as in Remark 1 .

REMARK 4. From the proof of Theorem 2 we obtain

$$
\begin{aligned}
& \frac{\partial v}{\partial x}(x, y)+\int_{0}^{y} l(x, z) d z v(x, y) \leq \frac{\partial w}{\partial x}(x, y)+\int_{0}^{y} l(x, z) d z w(x, y) \quad \text { a.e. on } I, \\
& \frac{\partial v}{\partial y}(x, y)+\int_{0}^{x} l(z, y) d z v(x, y) \leq \frac{\partial w}{\partial y}(x, y)+\int_{0}^{x} l(z, y) d z w(x, y) \quad \text { a.e. on } I .
\end{aligned}
$$

In [4] we discussed Theorems 1 and 2 for hyperbolic equations, in the case where $f$ was independent of $\partial u / \partial x, \partial u / \partial y$. Note that the assumptions in [4] are similar to those given above. Of course, in the present paper we have additional conditions in Theorems 1 and 2 on the first partial derivatives of $u, v$ and $w$. Moreover, in [4] we assume only that $c_{i j} \geq 0$ a.e. on $I$ for $i \neq j$, while in the present paper we need a stronger assumption that $c_{i j}(x, y) \geq 2 \int_{0}^{x} l(z, y) d z \int_{0}^{y} l(x, z) d z$ a.e. on $I$ for $i \neq j$. Now, we give an example which demonstrates that in Theorem 1 the assumption $c_{i j} \geq 0$ a.e. on $I$ for $i \neq j$ is not sufficient. 
EXAMPLE 2. We consider the system of inequalities

$$
\begin{cases}\frac{\partial^{2} u_{1}}{\partial x \partial y}(x, y) \geq \frac{2}{\sqrt{x y}} u_{1}(x, y)-\sqrt{\frac{x}{y} \frac{\partial u_{1}}{\partial x}(x, y)+x \frac{\partial u_{2}}{\partial x}(x, y)} & \text { a.e. in } I, \\ \frac{\partial^{2} u_{2}}{\partial x \partial y}(x, y) \geq(x y-1) u_{2}(x, y) & \text { a.e. in } I,\end{cases}
$$

where $I=[0, a] \times[0, a]$. We can verify that $u_{1}(x, y)=-x^{2} y^{2}$ and $u_{2}(x, y)=$ $e^{1-x y}$ satisfy (15). We have

$$
\begin{aligned}
& u_{1}(x, 0)=u_{1}(0, y)=\frac{\partial u_{1}}{\partial x}(x, 0)=\frac{\partial u_{1}}{\partial y}(0, y)=\frac{\partial u_{2}}{\partial x}(x, 0)=\frac{\partial u_{2}}{\partial y}(0, y)=0, \\
& u_{2}(x, 0)=u_{2}(0, y)=e .
\end{aligned}
$$

Moreover,

$$
\begin{gathered}
c_{11}(x, y)=\frac{2}{\sqrt{x y}}, \quad c_{12}(x, y)=c_{21}(x, y)=0, \quad c_{22}(x, y)=x y-1, \\
m_{11}(x, y)=-\sqrt{\frac{x}{y}}, \quad m_{12}(x, y)=4 x, \quad m_{21}(x, y)=m_{22}(x, y)=0, \\
n_{11}(x, y)=n_{12}(x, y)=n_{21}(x, y)=n_{22}(x, y)=0 .
\end{gathered}
$$

We can verify that for $a$ sufficiently small all assumptions of Theorem 1 are satisfied for $l(x, y)=2 / \sqrt[3]{x^{2} y^{2}}$ except $c_{i j}(x, y) \geq 2 \int_{0}^{x} l(z, y) d z \int_{0}^{y} l(x, z) d z$ for $i \neq j$. Since $u_{1}<0$ on $I \backslash I_{0}$, the assertion of Theorem 1 does not hold.

We can also note that the second inequality in $(15)$ shows that it is possible that $\frac{\partial u_{2}}{\partial x}(0, y)<0, \frac{\partial u_{2}}{\partial y}(x, 0)<0$ for $x \in(0, a], y \in(0, b]$ and $u_{2}>0$ on $I \backslash I_{0}$.

3. Weak inequalities for first order partial derivatives. We can find in [4] an example which shows that the assumptions in Theorem 1 are not sufficient to prove that $\partial u / \partial x, \partial u / \partial y \geq 0$ on $I$. Now we show that this can be proved under some additional assumptions.

TheOREm 3. Suppose that:

(I) For every $(x, y) \in I$ we have a linear map $P(x, y)$ from $C\left(D, \mathbb{R}^{k}\right)$ into $\mathbb{R}^{k}$ which is positive in the sense that $w \geq 0$ implies $P(x, y) w \geq 0$ for $(x, y) \in I$.

(II) $C(x, y)=\left(c_{i j}(x, y)\right)_{i, j=1}^{k}, M(x, y)=\left(m_{i j}(x, y)\right)_{i, j=1}^{k}, N(x, y)=$ $\left(n_{i j}(x, y)\right)_{i, j=1}^{k}$ are $k \times k$ matrices, where $c_{i j}, m_{i j}, n_{i j} \in L^{1}(I, \mathbb{R})$ 
and $c_{i j}(x, y) \geq 0, m_{i j}(x, y) \geq 0, n_{i j}(x, y) \geq 0$ a.e. in I. There is a function $l \in L^{1}(I, \mathbb{R})$ such that

$$
\begin{aligned}
& |P(x, y) w| \leq l(x, y)\|w\|_{0}, \quad c_{i j}(x, y) \leq l(x, y) \quad \text { a.e. in } I, \\
& m_{i j}(x, y) \leq \int_{0}^{x} l(z, y) d z, \quad n_{i j}(x, y) \leq \int_{0}^{y} l(x, z) d z \quad \text { a.e. in } I .
\end{aligned}
$$

(III) $u$ is a function absolutely continuous on $I$ and continuous on $I^{*}$; $\partial u / \partial x, \partial u / \partial y, \partial^{2} u / \partial x \partial y$ exist a.e. on $I ; \partial u / \partial x$ is continuous with respect to the second variable on $I$; and $\partial u / \partial y$ is continuous with respect to the first variable on I. Furthermore,

$$
\begin{gathered}
\frac{\partial^{2} u}{\partial x \partial y}(x, y) \geq C(x, y) u(x, y)+M(x, y) \frac{\partial u}{\partial x}(x, y) \\
+N(x, y) \frac{\partial u}{\partial y}(x, y)+P(x, y) u_{(x, y)} \quad \text { a.e. in } I, \\
u(x, y) \geq 0 \text { on } I_{0}, \quad \frac{\partial u}{\partial x}(x, 0) \geq 0 \quad \text { on }[0, a], \quad \frac{\partial u}{\partial y}(0, y) \geq 0 \quad \text { on }[0, b] .
\end{gathered}
$$

Then

$$
u(x, y) \geq 0, \quad \frac{\partial u}{\partial x}(x, y) \geq 0, \quad \frac{\partial u}{\partial y}(x, y) \geq 0 \quad \text { on } I .
$$

Proof. Let

$$
v^{1}(x, y)=e^{H(x, y)} \frac{\partial u}{\partial x}(x, y) \quad \text { and } \quad v^{2}(x, y)=e^{H(x, y)} \frac{\partial u}{\partial y}(x, y) .
$$

Then

$$
\begin{gathered}
\frac{\partial v^{1}}{\partial y}(x, y) \geq \int_{0}^{x} l(z, y) d z v^{1}(x, y)+e^{H(x, y)}\left\{C(x, y) u(x, y)+P(x, y) u_{(x, y)}\right\} \\
+M(x, y) v^{1}(x, y)+N(x, y) v^{2}(x, y) \\
\frac{\partial v^{2}}{\partial y}(x, y) \geq \int_{0}^{y} l(x, z) d z v^{2}(x, y)+e^{H(x, y)}\left\{C(x, y) u(x, y)+P(x, y) u_{(x, y)}\right\} \\
+M(x, y) v^{1}(x, y)+N(x, y) v^{2}(x, y)
\end{gathered}
$$

Define $\varrho: I^{*} \rightarrow \mathbb{R}, r: I^{*} \rightarrow \mathbb{R}^{k}$ by the formulas $\varrho(x, y)=e^{2 k H(x, y)+x+y}$, $r(x, y)=(\varrho(x, y), \ldots, \varrho(x, y))$ and let $p^{1}(x, y)=e^{H(x, y)} \frac{\partial r}{\partial x}(x, y), p^{2}(x, y)=$ $e^{H(x, y) \frac{\partial r}{\partial y}(x, y) \text {. Then }}$ 


$$
\begin{aligned}
p^{1}(x, y)= & \left\{2 k \int_{0}^{y} l(x, z) d z+1\right\} e^{H(x, y)} r(x, y), \\
p^{2}(x, y)= & \left\{2 k \int_{0}^{x} l(z, y) d z+1\right\} e^{H(x, y)} r(x, y), \\
\frac{\partial p^{1}}{\partial y}(x, y)= & \left\{2 k l(x, y)+2 k(2 k+1) \int_{0}^{x} l(z, y) d z \int_{0}^{y} l(x, z) d z\right. \\
& \left.+(2 k+1) \int_{0}^{x} l(z, y) d z+2 k \int_{0}^{x} l(x, z) d z+1\right\} e^{H(x, y)} r(x, y), \\
\frac{\partial p^{2}}{\partial x}(x, y)= & \left\{2 k l(x, y)+2 k(2 k+1) \int_{0}^{x} l(z, y) d z \int_{0}^{y} l(x, z) d z\right. \\
& \left.+2 k \int_{0}^{x} l(z, y) d z+(2 k+1) \int_{0}^{y} l(x, z) d z+1\right\} e^{H(x, y)} r(x, y) .
\end{aligned}
$$

Note that

$$
\begin{gathered}
0 \leq C(x, y) r(x, y) \leq k l(x, y) r(x, y), \quad\left|P(x, y) r_{(x, y)}\right| \leq l(x, y) r(x, y) \\
0 \leq M(x, y) p^{1}(x, y) \leq\left\{2 k^{2} \int_{0}^{x} l(z, y) d z \int_{0}^{y} l(x, z) d z\right. \\
\left.+k \int_{0}^{x} l(z, y) d z\right\} e^{H(x, y)} r(x, y) \\
0 \leq N(x, y) p^{2}(x, y) \leq\left\{2 k^{2} \int_{0}^{x} l(z, y) d z \int_{0}^{y} l(x, z) d z\right. \\
\left.+k \int_{0}^{y} l(x, z) d z\right\} e^{H(x, y)} r(x, y) .
\end{gathered}
$$

Thus

$$
\begin{aligned}
0 \leq & \int_{0}^{x} l(z, y) d z p^{1}(x, y)+e^{H(x, y)}\left\{C(x, y) r(x, y)+P(x, y) r_{(x, y)}\right\} \\
& +M(x, y) p^{1}(x, y)+N(x, y) p^{2}(x, y) \\
\leq & \left\{(n+1) l(x, y)+2 k(2 k+1) \int_{0}^{x} l(z, y) d z \int_{0}^{y} l(x, z) d z\right. \\
& \left.+k \int_{0}^{x} l(z, y) d z+(k+1) \int_{0}^{y} l(x, z) d z\right\} e^{H(x, y)} r(x, y) \\
\leq & \frac{\partial p^{1}}{\partial y}(x, y),
\end{aligned}
$$




$$
\begin{aligned}
0 \leq & \int_{0}^{y} l(x, z) d z p^{2}(x, y)+e^{H(x, y)}\left\{C(x, y) r(x, y)+P(x, y) r_{(x, y)}\right\} \\
& +M(x, y) p^{1}(x, y)+N(x, y) p^{2}(x, y) \\
\leq & \left\{(k+1) l(x, y)+2 k(2 k+1) \int_{0}^{x} l(z, y) d z \int_{0}^{y} l(x, z) d z\right. \\
& \left.+(k+1) \int_{0}^{x} l(z, y) d z+k \int_{0}^{y} l(x, z) d z\right\} e^{H(x, y)} r(x, y) \\
\leq & \frac{\partial p^{2}}{\partial y}(x, y) .
\end{aligned}
$$

Define

$$
\begin{aligned}
q_{\varepsilon}(x, y) & =u(x, y)+\varepsilon r(x, y) \\
v_{\varepsilon}^{1}(x, y) & =e^{H(x, y)} \frac{\partial q_{\varepsilon}}{\partial x}(x, y)=v^{1}(x, y)+\varepsilon p^{1}(x, y), \\
v_{\varepsilon}^{2}(x, y) & =e^{H(x, y)} \frac{\partial q_{\varepsilon}}{\partial y}(x, y)=v^{2}(x, y)+\varepsilon p^{2}(x, y) .
\end{aligned}
$$

From (16)-(19) and from the linearity of $P(x, y)$ we have

$$
\begin{aligned}
\frac{\partial v_{\varepsilon}^{1}}{\partial y}(x, y) \geq & \int_{0}^{x} l(z, y) d z v_{\varepsilon}^{1}(x, y) \\
& +e^{H(x, y)}\left\{C(x, y) q_{\varepsilon}(x, y)+P(x, y)\left(q_{\varepsilon}\right)_{(x, y)}\right\} \\
& +M(x, y) p^{1}(x, y)+N(x, y) p^{2}(x, y), \\
\frac{\partial v_{\varepsilon}^{2}}{\partial x}(x, y) \geq & \int_{0}^{y} l(x, z) d z v_{\varepsilon}^{2}(x, y) \\
& +e^{H(x, y)}\left\{C(x, y) q_{\varepsilon}(x, y)+P(x, y)\left(q_{\varepsilon}\right)_{(x, y)}\right\} \\
& +M(x, y) p^{1}(x, y)+N(x, y) p^{2}(x, y) .
\end{aligned}
$$

We see that there exists $c>0$ such that

$$
\begin{gathered}
q_{\varepsilon}(x, y)>0, \quad p^{1}(x, y)>0 \text { and } p^{2}(x, y)>0 \quad \text { on } I_{c}=I^{*} \backslash(c, a] \times(c, b], \\
v_{\varepsilon}^{1}(x, y)>0 \text { and } v_{\varepsilon}^{2}(x, y)>0 \quad \text { on }[0, c] \times[0, c] .
\end{gathered}
$$

Thus all terms on the right sides of (20) and (21) are nonnegative. Therefore $\partial v_{\varepsilon}^{1} / \partial y \geq 0$ and $\partial v_{\varepsilon}^{2} / \partial x \geq 0$ on $[0, c] \times[0, c]$. Thus $v_{\varepsilon}^{1}$ is nondecreasing with respect to the second variable and $v_{\varepsilon}^{2}$ is nondecreasing with respect to the first variable on $[0, c] \times[0, c]$. Therefore $q_{\varepsilon}$ is nondecreasing with respect to the first and second variables. This implies that $\partial v_{\varepsilon}^{1} / \partial y \geq 0$ and $\partial v_{\varepsilon}^{2} / \partial x \geq 0$ on $I_{c} \backslash\left(I^{*} \backslash I\right)$. Continuing we find that $\partial v_{\varepsilon}^{1} / \partial y \geq 0$ and $\partial v_{\varepsilon}^{2} / \partial x \geq 0$ on $I$. 
Therefore $v_{\varepsilon}^{1}>0$ and $v_{\varepsilon}^{2}>0$ on $I$. From the definitions of $v_{\varepsilon}^{1}$ and $v_{\varepsilon}^{2}$ we know that $\partial q_{\varepsilon} / \partial x>0$ and $\partial q_{\varepsilon} / \partial y>0$. Letting $\varepsilon \rightarrow 0^{+}$we get $\partial u / \partial x \geq 0$ and $\partial u / \partial y \geq 0$ on $I$. Of course this implies $u \geq 0$ on $I$.

TheOREM 4. Suppose that:

(I) The function $f: I \times C\left(D, \mathbb{R}^{k}\right) \times \mathbb{R}^{k} \times \mathbb{R}^{k} \rightarrow \mathbb{R}^{k}$ of the variables $(x, y, \omega, \mu, \nu)$ is nondecreasing with respect to $\omega, \mu$ and $\nu$.

(II) For each $A>0$ there exists a function $l \in L^{1}(I, \mathbb{R})$ such that

$$
\begin{aligned}
& |f(x, y, \omega, \mu, \nu)-f(x, y, \bar{\omega}, \bar{\mu}, \bar{\nu})| \\
& \quad \leq l(x, y)\|\omega-\bar{\omega}\|_{0}+\int_{0}^{x} l(z, y) d z|\mu-\bar{\mu}|+\int_{0}^{y} l(x, z) d z|\nu-\bar{\nu}|
\end{aligned}
$$

for $\|\omega\|_{0},\|\bar{\omega}\|_{0} \leq A$.

(III) $w, v$ are functions absolutely continuous on I and continuous on $I^{*}$; $\partial w / \partial x, \partial v / \partial x, \partial w / \partial y, \partial v / \partial y, \partial^{2} w / \partial x \partial y, \partial^{2} v / \partial x \partial y$ exist a.e. on $I ; \partial w / \partial x, \partial v / \partial x$ are continuous with respect to the second variable on $I$; and $\partial w / \partial y, \partial v / \partial y$ are continuous with respect to the first variable on $I$. Furthermore,

$$
\begin{aligned}
& \frac{\partial^{2} v}{\partial x \partial y}(x, y) \leq f\left(x, y, v_{(x, y)}, \frac{\partial v}{\partial x}(x, y), \frac{\partial v}{\partial y}(x, y)\right) \quad \text { and } \\
& \frac{\partial^{2} w}{\partial x \partial y}(x, y) \geq f\left(x, y, u_{(x, y)}, \frac{\partial w}{\partial x}(x, y), \frac{\partial w}{\partial y}(x, y)\right) \quad \text { a.e. in } I, \\
& v(x, y) \leq w(x, y) \quad \text { on } I_{0}, \\
& \frac{\partial v}{\partial x}(x, 0) \leq \frac{\partial w}{\partial x}(x, 0) \quad \text { on }[0, a], \quad \frac{\partial v}{\partial y}(0, y) \leq \frac{\partial w}{\partial y}(0, y) \quad \text { on }[0, b] .
\end{aligned}
$$

Then

$$
v(x, y) \leq w(x, y), \frac{\partial v}{\partial x}(x, y) \leq \frac{\partial w}{\partial x}(x, y), \frac{\partial v}{\partial y}(x, y) \leq \frac{\partial w}{\partial y}(x, y) \quad \text { on } I .
$$

Proof. Let $|v(x, y)|,|w(x, y)| \leq A-1$ for all $(x, y) \in I$ and $l$ be the function in condition (II) corresponding to $A$, and define

$$
\begin{aligned}
& V^{1}(x, y)=e^{H(x, y)} \frac{\partial v}{\partial x}(x, y), \quad W^{1}(x, y)=e^{H(x, y)} \frac{\partial w}{\partial x}(x, y), \\
& V^{2}(x, y)=e^{H(x, y)} \frac{\partial v}{\partial y}(x, y), \quad W^{2}(x, y)=e^{H(x, y)} \frac{\partial w}{\partial y}(x, y) .
\end{aligned}
$$

Then

$$
\begin{array}{ll}
\frac{\partial V^{1}}{\partial y}(x, y) \leq e^{H(x, y)}\left(G^{1} v\right)(x, y), & \frac{\partial W^{1}}{\partial y}(x, y) \geq e^{H(x, y)}\left(G^{1} w\right)(x, y), \\
\frac{\partial V^{2}}{\partial x}(x, y) \leq e^{H(x, y)}\left(G^{2} v\right)(x, y), & \frac{\partial W^{2}}{\partial y}(x, y) \geq e^{H(x, y)}\left(G^{2} w\right)(x, y),
\end{array}
$$


where

$$
\begin{gathered}
\left(G^{i} v\right)(x, y)=g^{i}\left(x, y, v_{(x, y)}, \frac{\partial v}{\partial x}(x, y), \frac{\partial v}{\partial y}(x, y), V^{i}(x, y)\right) \quad \text { for } i \in\{1,2\} \\
g^{1}(x, y, \omega, \mu, \nu, \sigma)=f(x, y, \omega, \mu, \nu)+e^{-H(x, y)} \int_{0}^{x} l(z, y) d z \sigma \\
g^{2}(x, y, \omega, \mu, \nu, \sigma)=f(x, y, \omega, \mu, \nu)+e^{-H(x, y)} \int_{0}^{y} l(x, z) d z \sigma
\end{gathered}
$$

and $(G w)(x, y)$ is defined by a similar formula to $(G v)(x, y)$. It is easily seen that $g^{i}$ are nondecreasing with respect to $\omega, \mu, \nu$ and $\sigma$.

Define $\varrho: I^{*} \rightarrow \mathbb{R}$ by $\varrho(x, y)=e^{2 H(x, y)+x+y}$ and $r, R^{1}, R^{2}: I^{*} \rightarrow \mathbb{R}^{k}$ by $r(x, y)=(\varrho(x, y), \ldots, \varrho(x, y)), R^{1}(x, y)=e^{H(x, y)} \frac{\partial r}{\partial x}(x, y)$ and $R^{2}(x, y)=$ $e^{H(x, y)} \frac{\partial r}{\partial y}(x, y)$. Defining $w_{\varepsilon}(x, y)=w(x, y)+\varepsilon r(x, y), W_{\varepsilon}^{i}(x, y)=W^{i}(x, y)+$ $\varepsilon R^{i}(x, y)$, where $i \in\{1,2\}$, we have

$$
\begin{aligned}
& \frac{\partial W_{\varepsilon}^{1}}{\partial y}(x, y) \geq e^{H(x, y)}\left(G^{1} w\right)(x, y) \\
& +\varepsilon e^{H(x, y)}\left\{l(x, y)+6 \int_{0}^{x} l(z, y) d z \int_{0}^{y} l(x, z) d z\right. \\
& \left.+3 \int_{0}^{x} l(z, y) d z+2 \int_{0}^{y} l(x, z) d z+1\right\} r(x, y), \\
& \frac{\partial W_{\varepsilon}^{2}}{\partial x}(x, y) \geq e^{H(x, y)}\left(G^{2} w\right)(x, y) \\
& +\varepsilon e^{H(x, y)}\left\{l(x, y)+6 \int_{0}^{x} l(z, y) d z \int_{0}^{y} l(x, z) d z\right. \\
& \left.+2 \int_{0}^{x} l(z, y) d z+3 \int_{0}^{y} l(x, z) d z+1\right\} r(x, y) .
\end{aligned}
$$

Moreover, we can choose $\varepsilon>0$ so small that $\left|w_{\varepsilon}(x, y)\right| \leq A$. From the Lipschitz condition for $f$ we have

$$
\begin{aligned}
\left|G^{1} w_{\varepsilon}(x, y)-G^{1} w(x, y)\right| \leq & \varepsilon\left\{l(x, y)+6 \int_{0}^{x} l(z, y) d z \int_{0}^{y} l(x, z) d z\right. \\
& \left.+2 \int_{0}^{x} l(z, y) d z+\int_{0}^{y} l(x, z) d z\right\} \varrho(x, y),
\end{aligned}
$$




$$
\begin{aligned}
\left|G^{2} w_{\varepsilon}(x, y)-G^{2} w(x, y)\right| \leq & \varepsilon\left\{l(x, y)+6 \int_{0}^{x} l(z, y) d z \int_{0}^{y} l(x, z) d z\right. \\
& \left.+\int_{0}^{x} l(z, y) d z+2 \int_{0}^{y} l(x, z) d z\right\} \varrho(x, y) .
\end{aligned}
$$

From the above inequality we get

$$
\begin{aligned}
e^{H(x, y)}\left(G^{1} w_{\varepsilon}\right)(x, y) \leq & e^{H(x, y)}\left(G^{1} w\right)(x, y) \\
& +\varepsilon e^{H(x, y)}\left\{l(x, y)+6 \int_{0}^{x} l(z, y) d z \int_{0}^{y} l(x, z) d z\right. \\
& \left.+2 \int_{0}^{x} l(z, y) d z+\int_{0}^{y} l(x, z) d z\right\} r(x, y) \\
\leq & \frac{\partial W_{\varepsilon}^{1}}{\partial y}(x, y) .
\end{aligned}
$$

Similarly, we obtain

$$
e^{H(x, y)}\left(G^{2} w_{\varepsilon}\right)(x, y) \leq \frac{\partial W_{\varepsilon}^{2}}{\partial x}(x, y) .
$$

Note that

$$
\begin{array}{ll}
w_{\varepsilon}(x, y)>w(x, y) \geq v(x, y) & \text { on } I_{0}, \\
\frac{\partial w_{\varepsilon}}{\partial x}(x, 0)>\frac{\partial w}{\partial x}(x, 0) \geq \frac{\partial v}{\partial x}(x, 0) & \text { on }[0, a], \\
\frac{\partial w_{\varepsilon}}{\partial y}(0, y)>\frac{\partial w}{\partial y}(0, y) \geq \frac{\partial v}{\partial y}(0, y) & \text { on }[0, b] .
\end{array}
$$

Therefore there exists $c>0$ such that

$$
\begin{array}{ll}
w_{\varepsilon}(x, y)>v(x, y) & \text { on } I_{c}=I^{*} \backslash(c, a] \times(c, b], \\
\frac{\partial w_{\varepsilon}}{\partial x}(x, y)>\frac{\partial v}{\partial x}(x, y) & \text { on }[0, a] \times[0, c], \\
\frac{\partial w_{\varepsilon}}{\partial y}(x, y)>\frac{\partial v}{\partial y}(x, y) & \text { on }[0, c] \times[0, b] .
\end{array}
$$

From monotonicity of $G^{1}$ and $G^{2}$ on $(0, c] \times(0, c]$ we have

$$
\begin{aligned}
& \frac{\partial W_{\varepsilon}^{1}}{\partial y}(x, y) \geq e^{H(x, y)}\left(G^{1} w_{\varepsilon}\right)(x, y) \geq e^{H(x, y)}\left(G^{1} v\right)(x, y) \geq \frac{\partial V^{1}}{\partial y}(x, y), \\
& \frac{\partial W_{\varepsilon}^{2}}{\partial x}(x, y) \geq e^{H(x, y)}\left(G^{2} w_{\varepsilon}\right)(x, y) \geq e^{H(x, y)}\left(G^{2} v\right)(x, y) \geq \frac{\partial V^{2}}{\partial x}(x, y),
\end{aligned}
$$

for $(x, y) \in(0, c] \times(0, c]$. Therefore $W_{\varepsilon}^{1}-V^{1}$ is nondecreasing with respect to the second variable, and $W_{\varepsilon}^{2}-V^{2}$ is nondecreasing with respect to the first variable on $(0, c] \times(0, c]$. Thus similarly to the proof of Theorem 2 we get 
$\partial w_{\varepsilon} / \partial x-\partial v / \partial x>0$ and $\partial w_{\varepsilon} / \partial y-\partial v / \partial y>0$ on $I$. Letting $\varepsilon \rightarrow 0$ we get

$$
\begin{array}{ll}
\frac{\partial w}{\partial x}(x, y) \geq \frac{\partial v}{\partial x}(x, y) & \text { on } I, \\
\frac{\partial w}{\partial y}(x, y) \geq \frac{\partial v}{\partial y}(x, y) & \text { on } I .
\end{array}
$$

From the above it is clear that $w \geq v$ on $I$.

4. Strong inequalities. In this section we will be concerned with strong inequalities. First, we present some definitions and assumptions. A measurable set $B \subset I$ is called dense at $\left(0^{+}, 0^{+}\right)$if $B \cap(0, \varepsilon) \times(0, \varepsilon)$ has positive measure for every $\varepsilon>0$. Let $g$ and $h$ be functions from $I$ into $\mathbb{R}^{k}$. We write $g(x, y)<h(x, y)$ at $\left(0^{+}, 0^{+}\right)$if $\{(x, y) \in I: g(x, y)<h(x, y)\}$ is dense at $\left(0^{+}, 0^{+}\right)$. In the one-dimensional case a measurable set $A \subset[0, a]$ is called dense at $0^{+}$if $A \cap(0, \varepsilon)$ has positive measure for every $\varepsilon>0$. We write $g(x, 0)<h(x, 0)$ at $0^{+}$if $\{x \in[0, a]: g(x, 0)<h(x, 0)\}$ is dense at $0^{+}$. Similarly, we define $g(0, y)<h(0, y)$ at $0^{+}$.

Assumption 1. $C=\left(c_{i j}\right)_{i, j=1}^{k}$ is such that for every pair $(\alpha, \beta)$ of disjoint nonempty sets with $\alpha \cup \beta=\{1, \ldots, k\}$ there are $i \in \alpha$ and $j \in \beta$ such that $c_{i j}(x, y)>2 \int_{0}^{x} l(z, y) d z \int_{0}^{y} l(x, z) d z$ at $\left(0^{+}, 0^{+}\right)$.

Assumption 2. $M=\left(m_{i j}\right)_{i, j=1}^{k}$ is such that for every pair $(\alpha, \beta)$ of disjoint nonempty sets with $\alpha \cup \beta=\{1, \ldots, k\}$ there are $i \in \alpha$ and $j \in \beta$ such that $m_{i j}(x, y)<\int_{0}^{x} l(z, y) d z$ at $\left(0^{+}, 0^{+}\right)$.

Assumption 3. $N=\left(n_{i j}\right)_{i, j=1}^{k}$ is such that for every pair $(\alpha, \beta)$ of disjoint nonempty sets with $\alpha \cup \beta=\{1, \ldots, k\}$ there are $i \in \alpha$ and $j \in \beta$ such that $n_{i j}(x, y)<\int_{0}^{y} l(x, z) d z$ at $\left(0^{+}, 0^{+}\right)$.

We write $P(x, y)$ in the matrix form $P_{i j}(x, y)$, where $P_{i j}(x, y)$ is a positive linear operator which acts on functions $w \in C\left(D, \mathbb{R}^{1}\right)$.

Definition 1. $P(x, y)$ is called irreducible at $\left(0^{+}, 0^{+}\right)$if for every pair $(\alpha, \beta)$ of disjoint nonempty index sets with $\alpha \cup \beta=\{1, \ldots, k\}$ there are $i \in \alpha$ and $j \in \beta$ such that $P_{i j}(x, y) w>0$ at $\left(0^{+}, 0^{+}\right)$for all $w \in C\left(D, \mathbb{R}^{1}\right)$ such that $w(s, t)>0$ on $D$.

TheOREM 5 (positivity). Under the assumptions of Theorem 1 we have $u>0$ on $\widetilde{I}$ under each of the following conditions:

(I) $u(x, 0)>0$ or $u(0, y)>0$ at $0^{+}$;

(II) $\frac{\partial^{2} u}{\partial x \partial y}(x, y)>C(x, y) u(x, y)+M(x, y) \frac{\partial u}{\partial x}(x, y)$

$+N(x, y) \frac{\partial u}{\partial y}(x, y)+P(x, y) u_{(x, y)}$ at $\left(0^{+}, 0^{+}\right) ;$ 
(III) $u(x, 0) \neq 0$ or $u(0, y) \neq 0$ at $0^{+}$and $C$ satisfies Assumption 1 ;

(IV) $u(x, 0) \neq 0$ or $u(0, y) \neq 0$ at $0^{+}$and $M$ satisfies Assumption 2;

(V) $u(x, 0) \neq 0$ or $u(0, y) \neq 0$ at $0^{+}$and $N$ satisfies Assumption 3 ;

(VI) $u(x, 0) \neq 0$ or $u(0, y) \neq 0$ at $0^{+}$and $P$ is irreducible.

Proof. It is easy to see that the assertion of Theorem 5 follows from conditions (I) and (II). In order to prove that this is also the case for conditions (III), (IV) and (V) we first assume that $\beta$ (as in Remark 1) is nonempty and we note that $\alpha$ is nonempty too. This is due to the fact that if $u(x, 0) \neq 0$ or $u(0, y) \neq 0$ at $0^{+}$then there is $d$ such that $u_{d}(x, y)>0$ on $\widetilde{I}$. Let $j \in \beta$ (that is, $u_{j}(x, y)=0$ at $\left.\left(0^{+}, 0^{+}\right)\right)$. Moreover, from Theorem 1 we have $u \geq 0$ on $I$. Therefore at $\left(0^{+}, 0^{+}\right)$we have

$$
\begin{aligned}
\frac{\partial^{2} u_{j}}{\partial x \partial y}(x, y) \geq & \sum_{i=1}^{k} c_{j i}(x, y) u_{i}(x, y) \\
& +\sum_{i=1}^{k} m_{j i}(x, y) \frac{\partial u_{i}}{\partial x}(x, y)+\sum_{i=1}^{k} n_{j i}(x, y) \frac{\partial u_{i}}{\partial y}(x, y) \\
\geq & \sum_{i=1}^{k} c_{j i}(x, y) u_{i}(x, y)-\int_{0}^{y} l(x, z) d z \sum_{i=1}^{k} m_{j i}(x, y) u_{i}(x, y) \\
& -\int_{0}^{x} l(z, y) d z \sum_{i=1}^{k} n_{j i}(x, y) u_{i}(x, y) \\
= & \sum_{1 \leq i \leq k} c_{j i}(x, y) u_{i}(x, y) \\
& -\int_{0}^{y} l(x, z) d z \sum_{\substack{1 \leq i \leq k \\
i \neq j}} m_{j i}(x, y) u_{i}(x, y) \\
& -\int_{0}^{x} l(z, y) d z \sum_{\substack{1 \leq i \leq k \\
i \neq j}} n_{j i}(x, y) u_{i}(x, y) .
\end{aligned}
$$

Suppose that condition (III) holds. Then from the estimates for $m_{i j}(x, y)$, $n_{i j}(x, y), c_{i j}(x, y)$ and from Assumption 1 we get

$$
\begin{aligned}
\frac{\partial^{2} u_{j}}{\partial x \partial y}(x, y) & \geq \sum_{\substack{1 \leq i \leq k \\
i \neq j}}\left[c_{j i}(x, y)-2 \int_{0}^{x} l(z, y) d z \int_{0}^{y} l(x, z) d z\right] u_{i}(x, y) \\
& \geq\left[c_{j d}(x, y)-2 \int_{0}^{x} l(z, y) d z \int_{0}^{y} l(x, z) d z\right] u_{d}(x, y)>0 .
\end{aligned}
$$


Thus $u_{j}(x, y)$ is increasing with respect to $x$ and $y$ at $\left(0^{+}, 0^{+}\right)$and this contradicts the fact that $j \in \beta$.

Analogously, if we assume condition (IV) we get

$$
\begin{aligned}
\frac{\partial^{2} u_{j}}{\partial x \partial y}(x, y) \geq & \sum_{\substack{1 \leq i \leq k \\
i \neq j}}\left[2 \int_{0}^{x} l(z, y) d z \int_{0}^{y} l(x, z) d z-\int_{0}^{y} l(x, z) d z m_{j i}(x, y)\right. \\
& \left.-\int_{0}^{x} l(z, y) d z \int_{0}^{y} l(x, z) d z\right] u_{i}(x, y) \\
\geq & {\left[\int_{0}^{x} l(z, y) d z \int_{0}^{y} l(x, z) d z-\int_{0}^{y} l(x, z) d z m_{j d}(x, y)\right] u_{d}(x, y)>0 . }
\end{aligned}
$$

Thus $u_{j}(x, y)$ is increasing with respect to $x$ and $y$ at $\left(0^{+}, 0^{+}\right)$, contrary to $j \in \beta$.

The proof is similar if we assume condition (V) instead of (IV).

It is easily seen that if we assume (VI) and if there exist $j \in \beta$ and $d \in \alpha$ then $\frac{\partial^{2} u_{j}}{\partial x \partial y}(x, y) \geq P_{j k}(x, y) u_{d}(x, y)>0$ at $\left(0^{+}, 0^{+}\right)$, contrary to $j \in \beta$.

Assumption 4. The function $f$ is such that for each pair $(\alpha, \beta)$ of nonempty disjoint index sets such that $\alpha \cup \beta=\{1, \ldots, k\}$ there exist $i \in \alpha$ and $j \in \beta$ such that for some $c>0$ and for all $(x, y) \in(0, c] \times(0, c]$ we have

$$
\begin{aligned}
f_{j}(x, y, \omega, \eta, \mu, \nu)< & f_{j}\left(x, y, \omega+\theta_{(x, y)} e_{i}, \eta+\theta(x, y) e_{i}, \mu, \nu\right) \\
& -2 \int_{0}^{x} l(z, y) d z \int_{0}^{y} l(x, z) d z|\theta(x, y)|,
\end{aligned}
$$

where $\theta(x, y) \in C\left(I^{*}, \mathbb{R}\right)$ is zero on $I_{0}$ and positive on $\widetilde{I}$.

TheOREM 6 (strong inequalities). Under the assumptions of Theorem 2 we have $v<w$ on $\widetilde{I}$ under each of the following conditions:

(I) $v(x, 0)<w(x, 0)$ or $v(0, y)<w(0, y)$ at $0^{+}$;

(II) $f\left(x, y, v_{(x, y)}, v(x, y), \frac{\partial v}{\partial x}(x, y), \frac{\partial v}{\partial y}(x, y)\right)$ $<f\left(x, y, w_{(x, y)}, w(x, y), \frac{\partial w}{\partial x}(x, y), \frac{\partial w}{\partial y}(x, y)\right)$ at $\left(0^{+}, 0^{+}\right) ;$

(III) $v(x, 0) \neq w(x, 0)$ or $v(0, y) \neq w(0, y)$ at $0^{+}$and $f$ satisfies Assumption 4 .

Proof. It is easy to see that the assertion follows from conditions (I) and (II). Suppose that (III) holds. Let $\alpha$ and $\beta$ be as in Remark 3. Thus $v_{i}(x, y)<$ $w_{i}(x, y)$ for $i \in \alpha$, and $v_{j}(x, y)=w_{j}(x, y)$ on $A_{j}$ and $v_{j}(x, y)<w_{j}(x, y)$ on $B_{j}$ for $j \in \beta$. Note that there exists $c>0$ such that $[0, c) \times[0, c) \subset A_{j}$. Indeed, 
if $v(x, y)<w(x, y)$ on $[0, c) \times[0, c)$ for some $c>0$ then $v(x, y)<w(x, y)$ on $\widetilde{I}$. Therefore there exist $\widetilde{c}>0$ and $A_{\widetilde{c}}=[0, \widetilde{c}) \times[0, \widetilde{c})$ such that $A_{\widetilde{c}} \subset A_{j}$ for each $j \in \beta$. We note that $\alpha$ is nonempty, because from $v(x, 0) \neq w(x, 0)$ or $v(0, y) \neq w(0, y)$ at $0^{+}$it follows that there exists $i$ such that $v_{i}(x, y)<$ $w_{i}(x, y)$ on $\widetilde{I}$. Assume that $\beta$ is nonempty. Choose $\left(x_{0}, y_{0}\right) \in A_{\widetilde{c}}$. Choose $d \in \alpha$ such that

$$
\begin{aligned}
\left|w_{d}\left(x_{0}, y_{0}\right)-v_{d}\left(x_{0}, y_{0}\right)\right| & =\max \left\{\left|w_{i}\left(x_{0}, y_{0}\right)-v_{i}\left(x_{0}, y_{0}\right)\right|: i \in \alpha\right\} \\
& =\max \left\{\left|w_{i}\left(x_{0}, y_{0}\right)-v_{i}\left(x_{0}, y_{0}\right)\right|: i \in \alpha \cup \beta\right\} .
\end{aligned}
$$

Then

$$
\begin{aligned}
& f^{\beta}\left(x_{0}, y_{0}, w_{\left(x_{0}, y_{0}\right)}, w\left(x_{0}, y_{0}\right), \frac{\partial w}{\partial x}\left(x_{0}, y_{0}\right), \frac{\partial w}{\partial y}\left(x_{0}, y_{0}\right)\right) \leq \frac{\partial^{2} w^{\beta}}{\partial x \partial y}\left(x_{0}, y_{0}\right) \\
= & \frac{\partial^{2} v^{\beta}}{\partial x \partial y}\left(x_{0}, y_{0}\right) \leq f^{\beta}\left(x_{0}, y_{0}, v_{\left(x_{0}, y_{0}\right)}, v\left(x_{0}, y_{0}\right), \frac{\partial v}{\partial x}\left(x_{0}, y_{0}\right), \frac{\partial v}{\partial y}\left(x_{0}, y_{0}\right)\right) .
\end{aligned}
$$

If $\theta(x, y)=w_{d}(x, y)-v_{d}(x, y)$ then from the assumption we have

$$
\begin{aligned}
& f^{\beta}\left(x_{0}, y_{0}, v_{\left(x_{0}, y_{0}\right)}, v\left(x_{0}, y_{0}\right), \frac{\partial v}{\partial x}\left(x_{0}, y_{0}\right), \frac{\partial v}{\partial y}\left(x_{0}, y_{0}\right)\right) \\
& <f^{\beta}\left(x_{0}, y_{0},\left(v^{\{d\}^{\prime}}, w^{\{d\}}\right)_{\left(x_{0}, y_{0}\right)},\left(v^{\{d\}^{\prime}}, w^{\{d\}}\right)\left(x_{0}, y_{0}\right), \frac{\partial v}{\partial x}\left(x_{0}, y_{0}\right), \frac{\partial v}{\partial y}\left(x_{0}, y_{0}\right)\right) \\
& \quad-2 \int_{0}^{x_{0}} l\left(z, y_{0}\right) d z \int_{0}^{y_{0}} l\left(x_{0}, z\right) d z\left|w_{d}\left(x_{0}, y_{0}\right)-v_{d}\left(x_{0}, y_{0}\right)\right| .
\end{aligned}
$$

Let $T, T^{\prime}, R, R^{\prime}$ be as in the proof of Theorem 2. Then from monotonicity of $f$, quasimonotonicity of $f$ and the fact that $w^{\beta}\left(x_{0}, y_{0}\right)=v^{\beta}\left(x_{0}, y_{0}\right)$ and $w\left(x_{0}, y_{0}\right) \geq v\left(x_{0}, y_{0}\right)$ we have

$$
\begin{aligned}
& f^{\beta}\left(x_{0}, y_{0},\left(v^{\{d\}^{\prime}}, w^{\{d\}}\right)_{\left(x_{0}, y_{0}\right)},\left(v^{\{d\}^{\prime}}, w^{\{d\}}\right)\left(x_{0}, y_{0}\right), \frac{\partial v}{\partial x}\left(x_{0}, y_{0}\right), \frac{\partial v}{\partial y}\left(x_{0}, y_{0}\right)\right) \\
& \quad \leq f^{\beta}\left(x_{0}, y_{0}, w_{\left(x_{0}, y_{0}\right)}, w\left(x_{0}, y_{0}\right), \frac{\partial\left(w^{T}, v^{T^{\prime}}\right)}{\partial x}\left(x_{0}, y_{0}\right), \frac{\partial\left(w^{R}, v^{R^{\prime}}\right)}{\partial y}\left(x_{0}, y_{0}\right)\right) .
\end{aligned}
$$

From the Lipschitz condition of $f$ and the estimates

$$
\begin{aligned}
\left|\frac{\partial v_{i}}{\partial x}\left(x_{0}, y_{0}\right)-\frac{\partial w_{i}}{\partial x}\left(x_{0}, y_{0}\right)\right| & \leq \int_{0}^{y_{0}} l\left(x_{0}, z\right) d z\left(w_{i}\left(x_{0}, y_{0}\right)-v_{i}\left(x_{0}, y_{0}\right)\right), \\
\left|\frac{\partial v_{j}}{\partial y}\left(x_{0}, y_{0}\right)-\frac{\partial w_{j}}{\partial y}\left(x_{0}, y_{0}\right)\right| & \leq \int_{0}^{x_{0}} l\left(z, y_{0}\right) d z\left(w_{j}\left(x_{0}, y_{0}\right)-v_{j}\left(x_{0}, y_{0}\right)\right),
\end{aligned}
$$


for $i \in T^{\prime}, j \in R^{\prime}$, we have

$$
\begin{aligned}
& f^{\beta}\left(x_{0}, y_{0}, w_{\left(x_{0}, y_{0}\right)}, w\left(x_{0}, y_{0}\right), \frac{\partial\left(w^{T}, v^{T^{\prime}}\right)}{\partial x}\left(x_{0}, y_{0}\right), \frac{\partial\left(w^{R}, v^{R^{\prime}}\right)}{\partial y}\left(x_{0}, y_{0}\right)\right) \\
& \leq f^{\beta}\left(x_{0}, y_{0}, w_{\left(x_{0}, y_{0}\right)}, w\left(x_{0}, y_{0}\right), \frac{\partial w}{\partial x}\left(x_{0}, y_{0}\right), \frac{\partial w}{\partial y}\left(x_{0}, y_{0}\right)\right) \\
& +\int_{0}^{x_{0}} l\left(z, y_{0}\right) d z \int_{0}^{y_{0}} l\left(x_{0}, z\right) d z\left[\left|w\left(x_{0}, y_{0}\right)-v\left(x_{0}, y_{0}\right)\right|_{T^{\prime}}+\left|w\left(x_{0}, y_{0}\right)-v\left(x_{0}, y_{0}\right)\right|_{R^{\prime}}\right]
\end{aligned}
$$

From the above inequalities and the definition of $d$ we get

$$
\begin{aligned}
& f^{\beta}\left(x_{0}, y_{0}, v_{\left(x_{0}, y_{0}\right)}, v\left(x_{0}, y_{0}\right), \frac{\partial v}{\partial x}\left(x_{0}, y_{0}\right), \frac{\partial v}{\partial y}\left(x_{0}, y_{0}\right)\right) \\
& \quad<f^{\beta}\left(x_{0}, y_{0}, w_{\left(x_{0}, y_{0}\right)}, w\left(x_{0}, y_{0}\right), \frac{\partial w}{\partial x}\left(x_{0}, y_{0}\right), \frac{\partial w}{\partial y}\left(x_{0}, y_{0}\right)\right) .
\end{aligned}
$$

Thus (22) and (23) give a contradiction. Therefore $\beta$ is an empty set.

REMARK 5. We note that for classical solutions of differential inequalities, first a theorem about strong inequalities is proved. Next using this theorem and an extra condition (for example the Lipschitz condition on $f$ ) we prove a theorem about weak inequalities. In this paper we see that in the case of Carathéodory solutions, first a theorem about weak inequalities is proved. Next using this theorem and an extra condition (for example see Assumption 4) we prove a theorem about strong inequalities.

\section{References}

[1] S. Brzychczy and J. Janus, Monotone iterative methods for nonlinear integro-differential hyperbolic equations, Univ. Iagell. Acta Math. 37 (1999), 246-261.

[2] T. Człapiński, Iterative methods for Darboux problem for partial functional differential equations, J. Inequal. Appl. 4 (1998), 141-161.

[3] M. Hirsch, Systems of differential equations that are competitive or cooperative, II: convergence almost everywhere, SIAM J. Math. Anal. 16 (1985), 423-439.

[4] A. Karpowicz, The Darboux problem for hyperbolic functional differential equations and inequalities in the sense of Carathéodory, to appear.

[5] V. Lakshmikantham and S. Leela, Differential and Integral Inequalities, Vols. I, II, Academic Press, New York, 1969.

[6] J. Szarski, Differential Inequalities, PWN, Warszawa, 1967.

[7] W. Walter, On strongly monotone flows, Ann. Polon. Math. 66 (1997), 269-274. 
A. Karpowicz

[8] W. Walter, Ordinary functional differential equations and inequalities in the sense of Carathéodory, Appl. Anal. 70 (1998), 85-95.

[9] —, Differential and Integral Inequalities, Springer, 1970.

Institute of Mathematics

University of Gdańsk

Wit Stwosz St. 57

80-952 Gdańsk, Poland

E-mail: akarpowi@math.univ.gda.pl

Received 28.11.2007

and in final form 3.3.2008 